Z Rheumatol $2021 \cdot 80: 285-287$

https://doi.org/10.1007/s00393-021-00986-5

Online publiziert: 15. März 2021

(c) Springer Medizin Verlag GmbH, ein Teil von Springer Nature 2021
Redaktion "Zeitschrift für Rheumatologie“

Springer Medizin Verlag, Heidelberg, Deutschland

\title{
Erratum zu: Die Zeitschrift für Rheumatologie dankt den Gutachtern 2020
}

Erratum zu:

10.1007/s00393-020-00954-5

Sehr geehrte Leserschaft, sehr geehrte Gutachter und Gutachterinnen,

manchmal passieren Dinge, die man unbedingt verhindern wollte. So wurde leider eine veraltete Namensliste publiziert. Für diesen Fehler entschuldigen wir uns und bitten die untenstehende korrigierte Nennung zu beachten.

Den Gutachtern und Gutachterinnen sprechen wir unseren Dank für ihre Unterstützung im Jahr 2020 und unsere Wertschätzung aus.

Durch ihren Einsatz garantieren sie im Rahmen des Peer-Review-Verfahrens der eingereichten Manuskripte die hohe Qualität und klinische Relevanz der Zeitschrift für Rheumatologie.

\section{Die Redaktion}

Dr. Katinka Albrecht, Berlin

Prof. Dr. Daniel Aletaha, Wien

Dr. Tobias Alexander, Berlin

Prof. Dr. Alexander Kreuter,

Oberhausen

Prof. Dr. Rieke Alten, Berlin

Dr. Andreas Altenburg, Dessau

Dr. Ioana Andreica, Herne

Dr. Peer Aries, Hamburg

Prof. Dr. Martin Aringer, Dresden

Prof. Dr. Christoph Baerwald, Leipzig

PD Dr. Xenofon Baraliakos, Herne

Dr. Jutta Bauhammer, Baden-Baden

Prof. Dr. Heidemarie Becker, Münster

Prof. Dr. Frank Beil, Hamburg

Dr. Karolina Benesova, Heidelberg

Prof. Dr. Raoul Bergner, Ludwigshafen

PD Dr. Norman Best, Jena

Dr. Christoph Biehl, Gießen
Dr. Robert Biesen, Berlin

Prof. Dr. Norbert Blank, Heidelberg

Prof. Dr. Jürgen Braun, Herne

Dr. Hans-Peter Brezinschek, Graz

Prof. Dr. Christoph Brochhausen,

Regensburg

Prof. Dr. Harald Burkhardt, Frankfurt

a. M.

Prof. Dr. Gerd-Rüdiger Burmester,

Berlin

Prof. Dr. Frank Buttgereit, Berlin

Dr. Johanna Callhoff, Berlin

Prof. Dr. Hans-Dieter Carl, Nürnberg PD Dr. Jacqueline Detert, Berlin

Dr. Harald Dinges, Kusel

Dr. Dr. Gabriel Dischereit, Marburg

Prof. Dr. Oliver Distler, Zürich

Prof. Dr. Thomas Dörner, Berlin

Dr. Ulrich Drott, Frankfurt a. M.

PD Dr. Boris Ehrenstein, Bad Abbach

Prof. Dr. Wolfgang Eich, Heidelberg

Dr. Joachim-Michael Engel, Bad

Liebenwerda

Prof. Dr. Eugen Feist, Vogelsang/

Gommern
Dr. Martin Feuchtenberger, Altötting

Prof. Dr. Christoph Fiehn, Baden-

Baden

PD Dr. Rebecca Elise Fischer-Betz,

Düsseldorf

Dr. Johannes Flechtenmacher,

Karlsruhe

Prof. Dr. Martin Fleck, Bad Abbach

Prof. Dr. Dirk Föll, Münster

Prof. Dr. Frauke Foerger, Bern

Prof. Dr. Ruth Fritsch-Stork, Wien

Prof. Dr. Markus Gaubitz, Münster

Prof. Dr. Angela Gause, Bad Bramstedt

Dr. Martin Gehlen, Bad Pyrmont

André Golla, Halle-Wittenberg

Prof. Dr. Winfried Graninger, Graz

Prof. Dr. Dr. Joachim Grifka, Bad

Abbach

Prof. Dr. Erika Gromnica-Ihle, Berlin

Prof. Dr. Wolfgang L. Gross, Lübeck

PD Dr. Claudia Günther, Dresden

PD Dr. Hildrun Haibel, Berlin

Prof. Dr. Michael Hammer,

Sendenhorst

Prof. Dr. Peter Härle, Mainz

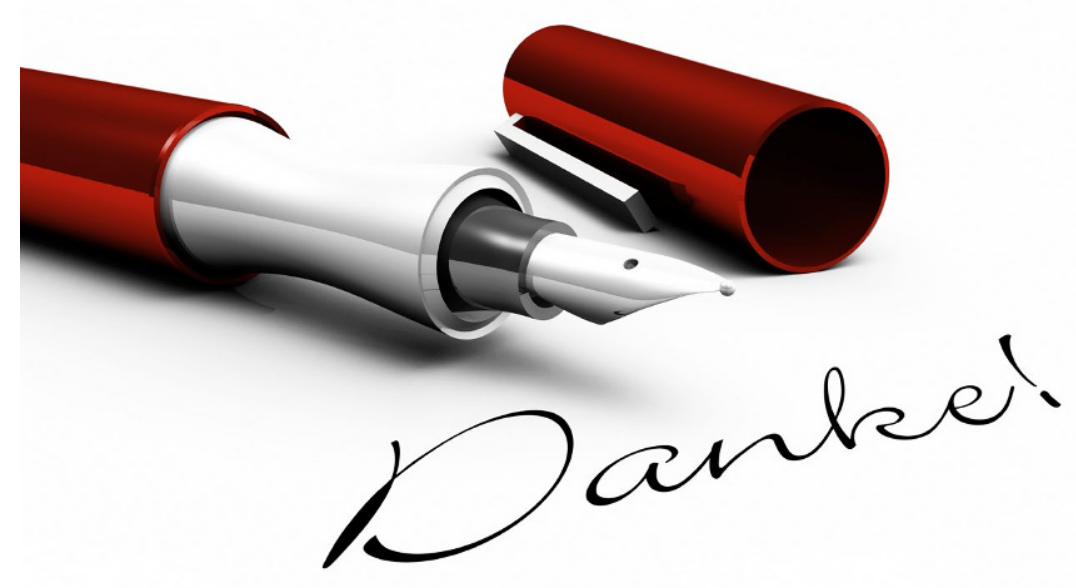


Prof. Dr. Wolfgang Hartung, Bad Abbach

Dr. Rebecca Hasseli, Bad Nauheim

Prof. Dr. Gert Hein, Weimar

Prof. Dr. Bernhard Hellmich,

Kirchheim

Prof. Dr. Joerg Henes, Tübingen

Dr. Walter Hermann, Bad Nauheim

Prof. Dr. Peter Herzer, München

Prof. Dr. Falk Hiepe, Berlin

Dr. Claas Hinze, Münster

Prof. Dr. Julia Holle, Neumünster

Prof. Dr. Gerd Horneff, St. Augustin

Prof. Dr. Bimba Hoyer, Kiel

Ole Hudowenz, Kiel

PD Dr. Dr. Axel Hueber, Bamberg

Prof. Hans-Iko Huppertz, Bremen

PD Dr. Christof Iking-Konert,

Hamburg

Dr. Sarah Johanssen, Berlin

Prof. Dr. Dr. Joachim Robert Kalden

$(\dagger)$, Erlangen

Dr. Ulrich Käßer, Gießen

Prof. Dr. Herbert Kellner, München

Prof. Dr. Gernot Keyßer, Halle (Saale)

Dr. David Kiefer, Herne

Dr. Philipp Klemm, Bad Nauheim

PD Dr. Arnd Kleyer, Erlangen

Prof. Dr. Christian Kneitz, Schwerin

Dr. Johannes Knitza, Erlangen

Dr. Peter Korsten, Göttingen

Prof. Dr. Ina Kötter, Hamburg

Dr. Marco Krasselt, Leipzig

Prof. Dr. Andreas Krause, Berlin

Prof. Dr. Veit Krenn, Trier

Prof. Dr. Joachim Kreuder, Gießen

Prof. Dr. Thomas Krieg, Köln

PD Dr. Gerhard Krönke, Erlangen

Prof. Dr. Klaus Krüger, München

Dr. Martin Krusche, Berlin

Prof. Dr. Jens Gert Kuipers, Bremen

Prof. Dr. Heinz-Jürgen Lakomek,

Minden

Prof. Dr. Peter Lamprecht, Lübeck

Prof. Dr. Uwe Lange, Gießen

PD Dr. Hans-Eckhard Langer,

Düsseldorf

Prof. Dr. Hartwig Lehmann, Gießen

Dr. Jan Leipe, Mannheim

Prof. Dr. Oliver Linhardt, München

Prof. Dr. Hanns-Martin Lorenz,

Heidelberg

Dr. Katharina Madlener, Bad Nauheim

Prof. Dr. Alfred Mahr, St. Gallen

Prof. Dr. Bernhard Manger, Erlangen

Prof. Dr. Ulrich Mansmann, München
Dr. Kerstin Mattukat, Halle-Wittenberg

Prof. Dr. Dirk Meyer-Olson, Bad

Pyrmont

Dr. Rudolf Mierau, Eschweiler

Prof. Dr. Kirsten Minden, Berlin

Prof. Dr. Frank Moosig, Neumünster

Dr. Johanna Mucke, Düsseldorf

PD Dr. Elena Neumann, Bad Nauheim

PD Dr. Sarah Ohrndorf, Berlin

Prof. Hans-Hartmut Peter, Freiburg

PD Dr. Alexander Pfeil, Jena

Prof. Dr. Denis Poddubnyy, Berlin

Prof. Dr. Georg Pongratz, Düsseldorf

Prof. Dr. Martina Prelog, Würzburg

Dr. Fabian Proft, Berlin

PD Dr. Andreas Ramming, Erlangen

PD Dr. Juergen Rech, Erlangen

PD Dr. Anne Regierer, Berlin

Prof. Dr. Stefan Rehart, Frankfurt a. M.

Prof. Dr. Eva Reinhold-Keller,

Hamburg

Prof. Dr. Jutta Richter, Düsseldorf

Prof. Dr. Gabriela Riemekasten, Lübeck

Prof Dr. Martin Rudwaleit, Bielefeld

Prof. Wolfgang Rüther, Hamburg

Dr. Petra Saar, Frankfurt a. M.

Prof. Bernd Salzberger, Regensburg

Dr. Oliver Sander, Düsseldorf

Dr. Martin Schäfer, Berlin

PD Dr. Valentin Schäfer, Bonn

Dr. Susanne Schalm, München

PD Dr. Anne Schänzer, Gießen

Dr. Dennis Scheicht, Köln

PD Dr. Marc Schmalzing, Würzburg

Dr. Tim Schmeiser, Wuppertal

Prof. Dr. Matthias Schneider,

Düsseldorf

Professor Dr. Benedikt Schoser,

München

Prof. Dr. Andreas Schwarting, Bad

Kreuznach

PD Dr. Philipp Sewerin, Düsseldorf

Prof. Dr. Mootaz Shousha, Bad Berka

Dr. Elise Siegert, Berlin

Dr. David Simon, Erlangen

Margarida Souto-Carneiro, Heidelberg

Dr. Susanna Späthling-Mestekemper,

München

Prof. Dr. Christof Specker, Essen

Prof. Tanja Stamm, Wien

Prof. Dr. Rainer Straub, Regensburg

Prof. Dr. Johannes Strunk, Köln

Dr. Paul Studenic, Wien

Dr. Bruno Stuhlmüller, Berlin

Prof. Dr. Norbert Suttorp, Berlin

Prof. Dr. Jens Thiel, Freiburg
Dr. Astrid Thiele, Wuppertal

Prof. Hans-Peter Tony, Würzburg

Dr. Henning Trawinski, Leipzig

PD Dr. Nils Venhoff, Freiburg

Prof. Dr. Peter Villiger, Bern

Prof. Dr. Reinhard Voll, Freiburg

Dr. Ulrich von Hinüber, Hildesheim

Prof. Johannes von Kempis, St. Gallen

PD Dr. Matthias Wahle, Augsburg

Prof. Dr. Siegfried Wassenberg,

Ratingen

Dr. Franziska Wiesent, München

Prof. Dr. Torsten Witte, Hannover

Prof. Dr. Jürgen Wollenhaupt,

Hamburg

Prof. Dr. Uwe Wollina, Dresden

Prof. Dr. Josef Zacher, Berlin

Dr. Michael Zaenker, Bernau

Prof. Dr. Henning Zeidler, Hannover

Prof. Dr. Fred Zepp, Mainz
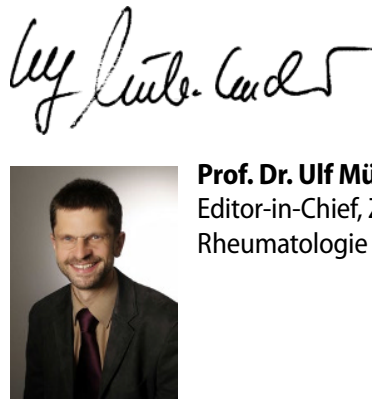

Prof. Dr. Ulf Müller-Ladner Editor-in-Chief, Zeitschrift für Rheumatologie
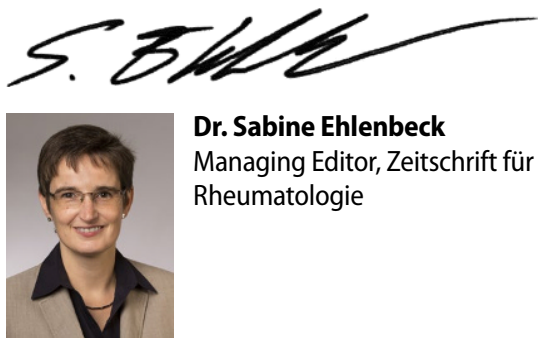

Dr. Sabine Ehlenbeck

Managing Editor, Zeitschrift für Rheumatologie

\section{Korrespondenzadresse}

Redaktion ,Zeitschrift für Rheumatologie“ Springer Medizin Verlag Heidelberg, Deutschland 
Hier steht eine Anzeige.

\section{算 Springer}

